\title{
PHARMACY BENEFIT MANAGEMENT, COST- EFFECTIVENESS ANALYSIS AND DRUG FORMULARY DECISIONS
}

\author{
HENRY GRABOWSKI ${ }^{*}$ and C. DANIEL MULLINS ${ }^{2}$ \\ 'Duke University, Department of Economics, Box 90097, Durham, NC 27708-0097, U.S.A. and \\ 'University of Maryland, MD, U.S.A.
}

\begin{abstract}
Pharmacy benefit management companies (PBMs) have evolved over the past decade in response to the increased demand for health care cost containment. Their activities include the implementation of drug formularies and the negotiation of rebates from manufacturers. Our analysis of this industry is based on interviews and materials provided by the top five ranked PBM companies which account for over $80 \%$ of beneficiaries covered within formulary plans. The formularies of these companies are relatively inclusive, but they are becoming more restrictive over time. At present the use of cost-effectiveness (C-E) studies in the formulary decisions of PBMs has been limited. In this regard, the surveyed PBMs emphasized that most C-E studies have not compared therapeutic substitutes in populations with characteristics that are similar to those of their clients. Pharmacy benefit management companies also have had strong incentives to focus narrowly on drug costs because they typically manage drug benefits on a "carved-out" basis. However, PBMs anticipate a growing future role in the integrated management of patient care (disease management) for certain high cost chronic diseases and conditions. All of the leading firms we surveyed have disease management programs in development. The importance of C-E studies to PBM decisions is expected to increase significantly as disease management programs are implemented. The data infrastructure inherent to the PBM industry and the increasing number of employees with advanced training in pharmacoeconomics will permit firms to perform their own internal C-E studies. They are also establishing various alliances and joint ventures with drug manufacturers, health maintenance organizations, and academic institutions to perform these analyses. The leading PBMs tend to favor active participation in the development of methodological approaches to C-E studies over government regulations such as those proposed by the FDA in 1995. $\mathrm{C} 1997 \mathrm{Else}$ vier Science Ltd
\end{abstract}

\section{INTRODUCTION}

The emergence of pharmacy benefit management companies (PBMs) constitutes a major structural change that has occurred in the distribution of pharmaceuticals during the 1990s. Pharmacy benefit management companies provide a variety of services designed to influence outpatient prescription drug usage and costs. It has been estimated that over $60 \%$ of employers utilized some form of PBM services in 1995 with coverage of 137 million beneficiaries (Health Strategies Group, 1995).

Pharmacy benefit management firms have evolved over the past decades from very diverse originse.g. claims processing firms, mail order pharmacies, and subsidiaries of insurance firms and managed

\footnotetext{
*Author for correspondence.

+The Catastrophic Health Care Act of 1988, which mandated outpatient prescription benefits for Medicare beneficiaries, was a major catalyst in the conversion of the retail pharmacies to on-line capabilities. While the Act was repealed prior to implementation of the outpatient prescription drug benefit, one of its key legacies was the ability of retail pharmacies to perform on-line claims processing and adjudication. This became a critical component underlying the rapid growth of PBMs during the 1990 s.
}

care organizations. A key factor that has fueled the industry's explosive growth in recent years is, of course, the increasing demand for cost control by health care payors. Also very important in the case of PBMs, however, was the development of on-line electronic data interchange capabilities at virtually all retail pharmacies by the end of the $1980 \mathrm{~s}$. $\dagger$

At the most basic level, PBMs perform electronic claims processing, provide a network of retail pharmacies and mail order pharmacy service, and offer pharmacy benefit plan design with patient cost sharing and other incentives. These activities generate cost savings to plan sponsors by reducing prescription claims processing charges, lowering ingredient and dispensing fees, and increasing patient co-payments.

Beyond these basic services, PBMs also engage in various activities designed to influence drug dispensing and prescribing. These activities include generic substitution, formularies, preferred drug lists, therapeutic interchange programs, treatment guidelines, drug utilization review and prior authorization. One of the main sources of cost savings to plan sponsors arises from the ability of PBMs to secure rebates from manufacturers for including their products on formularies and preferred drug lists. Arrangements 
Table 1. Leading PBM companies, June 1995

\begin{tabular}{lcc}
\hline Company & $\begin{array}{c}\text { Beneficiaries in } \\
\text { formulary plans } \\
\text { (millions) }\end{array}$ & $\begin{array}{c}\text { Total covered } \\
\text { beneficiaries (millions) }\end{array}$ \\
\hline PCS & 32 & 55 \\
Medco & 32 & 42 \\
Pharmacy Gold & 15 & 15 \\
Diversified & 12.9 & 12.9 \\
Value Health & 7.5 & 8.0 \\
Caremark & 5.2 & 28.0 \\
Advance Paradigm & 5.0 & 6.6 \\
Express Scripts & 4.8 & 6.9 \\
Diagnostek & 4.5 & 11.0 \\
Wellpoint & 2.0 & 5.0 \\
Less duplication & $(29.0)$ & $(66)$ \\
All other PBM & 2.0 & 13 \\
companies & & 137 \\
Industry total & 93.9 &
\end{tabular}

Source: Health Strategies Group (1995).

Notes: A covered beneficiary is an individual enrolled in a drug benefit plan managed by the PBM company; a beneficiary in a formulary plan is one enrolled in a drug benefit plan that has a formulary of recommended or approved drugs managed by the PBM company.

between PBMs and manufacturers increasingly take the form of performance-related contracts which tie the size of rebates to volume targets and other incentive-based milestones. Rebates have been a principal driver of the PBM industry during the 1990s.

The PBM industry is a very dynamic one with considerable entry and consolidation over the past five years. It has been estimated that over 40 firms provided PBM services to third parties on a forprofit basis in 1995 (U.S. General Accounting Office, 1995). Table 1 shows a list of the leading PBM firms in June 1995 with information on their covered beneficiaries in formulary plans, and their total covered beneficiaries. Because many beneficiaries are covered by two or more firms with differ-

*In the last two years, Merck acquired Medco. SmithKline Beecham acquired Diversified, and Lilly acquired PCS. These mergers have been somewhat controversial, spawning scrutiny by the FTC concerning the possibility of vertical foreclosure of rival pharmaceutical products and related practices. In this regard, Eli Lilly and PCS entered into a consent decree with the FTC to provide an open formulary option and to prevent information acquired by PCS on rival manufacturers competitive practices from being revealed to Lilly. Some of the competitive issues posed by these mergers have been recently considered by the U.S. General Accounting Office (1995).

fIt should be further noted that the fifth ranked firm, Value Health, acquired the ninth ranked one, Diagnostek after June 1995. The merged firm had 12.0 million individuals enrolled in formulary plans. The next ranked firm, Caremark, had only 5.2 million beneficiaries of this kind. Hence, given the large share held by the top five firms and the large gap between the fifth and sixth firm in terms of enrolled beneficiaries in formulary plans, there was a natural dividing line in selecting the sample of firms for the resource-intensive interview process. All of the top five firms agreed to participate, and we did not approach any other firms for personal interviews. ent services, one needs to take account of potential double counting in analyzing this industry. Even allowing for this fact, however, it is clear that the top 10 PBM firms account for a large share of covered beneficiaries (over 80\%). While the first part of this decade was characterized by considerable entry into the PBM business, the last few years have been characterized by considerable consolidation including vertical mergers between leading PBM and pharmaceutical firms.*

Competition in the PBM industry has centered around managing drug benefits on a carved-out basis, independent of other health care services and benefits. However, many PBM firms anticipate a growing future role in the management of patient care (disease management) for several high cost chronic diseases and conditions (e.g. asthma and diabetes). This would allow PBM firms to profit from the expected movement by managed care organizations toward a more coordinated systembased approach to health care problems. Of course, disease management requires the integration of medical data bases and a much broader perspective than PBMs have employed when managing drug costs on a carved-out basis.

The development of more sophisticated disease management capabilities also has been a major rationale offered for the mergers and alliances that have occurred between pharmaceutical firms and PBMs. Many of these joint efforts are initially focusing on high cost, chronic diseases such as asthma, diabetes and depression. These are diseases in which patient compliance and undertreatment are major issues.

In this paper, we are especially interested in analyzing how PBMs utilize cost-effectiveness studies in the management of drug benefits. Information for this analysis was obtained from several sources, including personal interviews at five leading PBMs. The five firms-PCS, Medco, Pharmacy Gold, Diversified and Value Health-are the five largest firms in terms of beneficiaries enrolled in formulary plans (Table 1). Together they account for over $80 \%$ of the beneficiaries enrolled in formularies. Hence, we felt this was the appropriate sample of firms to survey in terms of the use of C-E analysis in formulary decisions and related activities. $\dagger$ At each of these five surveyed companies, we spoke with individuals involved with pharmacy and therapeutic (P\&T) Committee decisions, outcome analyses, and business strategy. These firms also provided formularies, treatment guidelines and other relevant documents to us. This was supplemented by materials from some of the smaller PBMs and from third parties that compile data and information on the PBMs industry.

In the next section of the paper, we examine the nature of formularies and other interventions that PBMs currently utilize to influence drug prescription choices. The use of cost-effectiveness studies in 
Table 2. Estimated annual cost savings in the drug budget

\begin{tabular}{lc}
\hline \multicolumn{1}{c}{ PBM drug product selection activities } \\
\hline Generic substitution & $6-10 \%$ \\
Formularies (including & $5-15 \%$ \\
compliance measures) & \\
Concurrent drug utilization & $2-4 \%$ \\
review & $1-2 \%$ \\
Prior authorization & $14-31 \%$ \\
Total range &
\end{tabular}

Source: Industry publications and interviews with the five leading PBMs.

these activities has been limited. However, as the scope of PBM activities broadens over time to various disease management activities, the importance of cost-effectiveness studies to PBMs is expected to increase significantly. The third section of the paper considers these issues, as well as the role of government policy-makers like the U.S. Food and Drug Administration (FDA) in regulating cost-effectiveness claims made by manufacturers to PBM firms and other managed care organizations.

\section{FORMULARIES AND RELATED DRUG PRODUCT SELECTION ACTIVITIES}

In our interviews, we obtained estimates from the leading PBMs of the potential savings to the drug budget to payors, relative to an unmanaged plan, from PBM interventions designed to affect drug product selection. This information is presented in Table 2. These activities can produce estimated savings between 14 and $31 \%$ in a health plan's total drug expenditures $(25-40 \%$ when one also takes account of basic PBM activities like claims processing, pharmacies and mail order services, and benefit design). The major savings accrue from generic substitution and formularies, including various compliance measures.

\section{Generic substitution}

As shown in Table 2, generic substitution can save payors up to $10 \%$ of their total drug costs. In this regard, a managed plan can achieve generic utilization rates of $35-45 \%$, compared to rates of $15-20 \%$ for unmanaged plans. Generic substitution is typically encouraged through various benefit designs. A common feature is a two-tiered co-payment or co-insurance approach, with patients paying significantly less for generics than the branded product. Popular multiple-source products also may be subjected to a maximum allowable cost reimbursement policy set at the generic level. In addition, physicians that write DAWs disproportionately may be targeted in retrospective drug utilization review letters, including a qualitative analysis of how much patients could save through generic prescribing.

Pharmacy benefit management policies encouraging generic prescribing have become a major factor contributing to the more rapid erosion of branded drugs sales revenues when patents expire. Some
Table 3. Patented drugs experiencing initial generic competition 1989-1993

\begin{tabular}{lc}
\hline Cohort & $\begin{array}{c}\text { Generic market share (units) } \\
\text { Six months after entry }\end{array}$ \\
\hline $1989-1990$ & $31 \%$ \\
$1991-1992$ & $44 \%$ \\
1993 & $51 \%$ \\
\hline
\end{tabular}

Source: Grabowski and Vernon (1996).

representative results from a study by Grabowski and Vernon (1996) are presented in Table 3. As this table shows, the more recent cohorts of major drugs coming off patent since 1989 have experienced greater sales losses to generics. In this respect, the 1993 cohort lost over $50 \%$ of their market share (in units) during the first six months of generic competition, compared to $31 \%$ for the 1989 . 1990 cohort. Furthermore, the cohort of similar drugs coming off patent in the period 1984-1988 didn't experience market share losses of $50 \%$ to generics until more than two years after generic competition was introduced (Grabowski and Vernon, 1992).

The large number of commercially important drugs whose patents are scheduled to expire between now and the year 2001 will make generic substitution an important source of ongoing savings in drug benefit expenditures over future periods. One recent analysis indicated that drugs with 13 billion dollars in value are scheduled to come off patent between 1994 and the year 2001 (Treppell, 1994). Another analyst projects that generic dispensing could potentially account for $65 \%$ of all prescriptions by the end of the decade (Shaw, 1992).

\section{The formulary decision process}

Because PBMs have a set of clients that range from traditional indemnity plans to PPOs and more restrictive health maintenance organizational (HMO) forms, they have developed a spectrum of formulary instruments. These can be classified into open, preferred and closed formularies. Open formularies are merely lists of recommended drugs for which relative cost information is provided. Preferred formularies impose lower co-payments for drugs on the formulary. Closed formularies provide reimbursements only for drugs on the formulary.

It is up to the client firms to select how aggressive and restrictive they wish to be in their formulary interventions. A large share of the users of PBM services are employers that have evolved recentiy from fee-for-service to PPO or gatekeeper models of managed care. These plans usually elect, at least initially, an open formulary that gives a wide choice in prescription medicines. The main idea here is to educate physicians and patients on the differential costs of alternative medicines.

Employers then generally move over time to incorporate the economic incentives associated with a preferred drug formulary. These formularies pro- 
Table 4. Number of products on formulary selective cardiovascular classes

\begin{tabular}{lcccc}
\hline & Diuretics & ACE inhibitors & Beta blockers & Calcium channel blockers \\
\hline PBM 1 & 18 & 8 & 15 & 17 \\
PBM 2 & 15 & 7 & 14 & 17 \\
PBM 3 & 12 & 5 & 8 & 11 \\
PBM 4 & 15 & 7 & 10 & 14 \\
\hline
\end{tabular}

Source: Information supplied by surveyed PBM companies.

grams are generally complemented by retrospective drug utilization reviews and therapeutic interchange programs (where pharmacists call physicians in an effort to persuade them to switch prescriptions to formulary listings). Closed formularies, in which only listed drugs are reimbursed, are unusual in employer plans and are largely restricted to stafftype HMOs.*

A key decision for PBMs is which drugs should be included in their formularies. We found that PBMs typically employ a two-stage or bifurcated decision process in this situation. The initial evaluation of a new drug entity resides with the P\&T Committee. Some PBMs utilize outside experts for this purpose, while others rely on in-house staff. The key decision-makers on the P\&T Committee are physicians and clinical pharmacists. The focus of this committee is on a new drug's comparative efficacy and safety and its substitutability with existing drugs. Usually new drugs are evaluated within a short time interval around their approval by the FDA. All available information is utilized in preparing the dossier for the committee's review and action, including the FDA's approved labeling, published studies, and materials supplied by the drug manufacturer (Blissenbach, 1993).

The P\&T Committee decision leads to three possible outcomes. They can decide that a new drug must be included on the formulary (because it offers significant therapeutic advantages over existing drugs), $\dagger$ it must not be included on the formulary (because it has inferior clinical properties), or that it may be included on the formulary (because it is determined to be broadly interchangeable with existing drugs)

Many new drugs are part of an existing chemical class of compounds (e.g. ACE inhibitors, calcium

*In a survey of 200 medium and large employers with drug carve-out plans, by Health Strategies Group (1995), they found that $38 \%$ had formulary co-pay differentials and $14 \%$ had closed formularies.

tThe proposition that unique new drugs offering significant therapeutic advances will be approved for formulary inclusion was consistent with the information we reviewed. However, since these medicines are often expensive and lead to rising drug budgets, PBMs may recommend restrictive use provisions or prior authorization. Many biotech entities such as human growth hormone were included in the list of drugs recommended for prior authorization.

†Individual health care plans may opt for more restrictive formularies, but this is mainly done by HMO-type plan clients. channel blockers, etc.). If the P\&T Committee determines that a new drug is basically interchangeable with other drugs in its class, then issues of cost become important to the formulary decision. At this point, business decision-makers at the PBMs evaluate the new drug's cost relative to substitute therapies and become involved in the negotiation of rebates with manufacturers.

We found that the formularies of the leading PBMs are relatively inclusive. This is illustrated by the data in Table 4. This table shows the drugs on the formulary for different leading PBMs in several cardiovascular classes. It is noteworthy, for example, that the market for ACE inhibitors includes only eight drug products (seven distinct chemical entities), and that there are between five and eight drugs on the national PBM formularies of these five top PBM companies. $\ddagger$

The inclusive nature of current PBM formularies reflects a number of factors. First, physicians and patients historically have been used to little or no restrictions on prescription drugs in conventional indemnity plans. They are gradually becoming acclimated to the necessity of restrictions in choice in health care as managed care grows in scope and scale. Second, the pioneering or first-to-market products in a new therapeutic area have significant first-mover advantages with physicians. Later entrants in a class generally sell at lower prices, unless they offer important incremental therapeutic advantages. PBMs and other managed care entities have been a strong catalyst to the stimulation of price competition among drugs in major crowded therapeutic class (Grabowski, 1995). To date, however, PBMs have tended to add new lower priced drugs to their formularies, without deleting a corresponding number of rival brands in its class. Hence, rather than having only two or three drugs in a class like the ACE inhibitors, they have five to eight brands.

The PBM formularies list drugs by therapeutic class and provide relative cost indices on drugs deemed to be close substitutes. In particular, each product listed is accompanied by one to five (or sometimes six) dollar signs ( $\$$ to $\$ \$ \$ \$ \$$ ) to denote relative costs. Products that are available generically are given an asterisk or some special marking.

Table 5 provides an example of formulary listings for the ACE inhibitors class for the five PBMs in our survey. Most products in this class span a range of $\$ \$$ to $\$ \$ \$ \$$ with the newer products in the class having lowered costs than the established mar- 
Table 5. Formulary cost indices - ACE inhibitors

\begin{tabular}{|c|c|c|c|c|c|}
\hline & PBM 1 & РВM 2 & PBM 3 & PBM 4 & PBM 5 \\
\hline Monopril & $\$$ & $\$ \$$ & $\$ \$$ & $\$ \$$ & --- \\
\hline Lotensin & $\$ \$$ & $\$ \$$ & $\therefore$ & $\$ \$$ & $\$ S$ \\
\hline Altace & $\$ \$$ & SS & $\$ \$$ & $\$ \$$ & - \\
\hline Accupril & $\$ \$$ & $\cdots$ & $\$ S$ & $\$ \$$ & $\$ \$ \$$ \\
\hline Zestril & $\$ S \$$ & $\$ \$$ & $\$ \$$ & - & \\
\hline Prinivil & $\$ \$ S$ & $\$ \$$ & & $\$ \$$ & $\$ \$$ \\
\hline Vasotec & $\$ \$ \$ \$$ & $\$ \$ \$$ & $\cdots$ & $\$ \$ \$$ & $\$ \$ \$$ \\
\hline Capoten & $\$ \$ \$ \$$ & $\$ \$ \$$ & $\$ \$ \$ \$$ & $\$ \$ \$ \$$ & $\$ \$ \$ \$$ \\
\hline
\end{tabular}

Source: Information contained in PBM formularies for five surveyed companies.

ket leaders (Capoten and Vasotec). As expected, there is a high rank correlation in the relative cost indices of different PBM firms. Differences in cost indices reflect different rebate arrangements as well as differences in scaling.

The degree to which these relatively inclusive formularies can result in significant cost savings depends on their associated incentive and compliance measures. As discussed above, many plans include lower co-payments for formulary drugs. Other forms of compliance actions focus on physicians prescribing behavior through retrospective drug utilization evaluations (DUE). Under this approach, physicians that fall outside prescribing norms are subject to "alert letters" and possible financial penalties.

At the present time, there is a strong dynamic toward the development of more restrictive formularies (i.e. fewer drugs in a class) and stronger compliance measures for clients by PBMs.* Among other things, this reflects the fact that pharmaceutical manufacturers are willing to negotiate larger dis-

*All of the leading PBM firms in our survey indicated this to be the case. and it was generally borne out by a recent survey undertaken by the Health Strategies Group (1995). They found, for example, a growing use of economic incentives in carved-out employer-based formularies. While $38 \%$ of employers now use differential co-pay for formulary drugs, this is expected to grow to $51 \%$ in the near future.

†Beyond offering employer plans, the option of more restrictive, or even closed formularies, several PBMs have earmarked certain drugs in their formulary for priority status. PBMs then employ a variety of incentive measures to increase usage of these priority drugs, which in turn triggers larger rebates from the manufacturer. These measures can include therapeutic switch campaigns in which clinical pharmacists phone physicians. Physician behavior may also be influenced by the use of withhold pools and other financial incentives. Similarly, patients and retail pharmacists are also offered incentives to increase usage of the priority drugs in a given class.

$\ddagger$ There is evidence that government-run formularies utilized by certain states to control Medicaid drug costs resulted in significant cost shifting to other health care services (Moore and Newman, 1992). However, the decision-making process by which Medicaid programs approved new drugs for formularies (Grabowski, 1988) differs significantly from that which is employed by PBMs. Analysis of the effects of PBM formularies on cost shifting and patient outcomes remains an important issue for further work. counts in exchange for increased market share. $\dagger$ Staff HMOs have long recognized this fact and utilized it to obtain large cost savings in their drug expenditures.

The use of cost-effectiveness studies in formulary decisions

The use of pharmacoeconomic studies in PBM formulary decisions has been limited. PBM decision-makers that we interviewed provided several reservations concerning the applicability of existing pharmacoeconomic studies for their formulary decisions. First, there is the issue of the comparators used in these studies. There are few costeffectiveness (C-E) studies that compare closely substitutable products in the same class (e.g. one $\mathrm{ACE}$ inhibitor vs another). Rather, the comparison is typically with an older-type therapy or a placebo. Second, there is the issue of the population studied. Generally C-E studies have not specifically focused on the populations with characteristics served by the PBM's clients. Third, there are a number of perceived methodological issues that remain open with regard to $\mathrm{C}-\mathrm{E}$ studies (measurement of costs and benefits, discount rates, etc.). Fourth, there are perceived concerns about the objectivity of firm-sponsored C-E studies. Finally, some PBMs mentioned the need for better-educated sales representatives who would be able to address questions concerning C-E claims.

Given the lack of persuasive evidence from C-E studies showing clear differences among competing drugs in the same class, PBMs feel that their current decision approach to the evaluation of a product's cost-effectiveness is justified. In particular, in their view, the cost-effectiveness of one ACE inhibitor vs another turns primarily on differences in drug costs (including rebates). They believe that drug formularies constructed on this basis can yield significant savings to a plan's drug expenditures without leading to changes in health outcomes, problems of cost shifting, or losses in quality of life for patients. $\ddagger$

Table 6 presents a list of what PBMs would like to see in C-E studies to make the findings more applicable to their formulary decisions. These points address the various perceived limitations discussed above. Beyond wishing to see timely, head-to-head comparisons among market leaders in relevant 
Table 6. What PBMs would like to see in C-E studies

- Head-to-head comparisons among market leaders
- Studies performed on relevant populations
- Timely availability of information
- Independent sponsorship or no-strings funding
- Publication in leading peer-reviewed journals
- More sophisticated drug representatives who can discuss nuances
of a study

Source: Interviews with five surveyed PBMs.

populations, our surveyed firms also would like to see independent sponsorship or no-string funding to insure objectivity of these studies. However, many individuals indicated that this is an ideal but possibly unrealistic expectation, given that the main financial incentives to do the studies now reside with the manufacturers. Publication in highly regarded peer journals is viewed as an acceptable second best solution in most instances.

We found it significant that while PBMs were concerned about the objectivity of studies financed by manufacturers, there was little support for formal FDA regulation on the $\mathrm{C}-\mathrm{E}$ claims made by manufacturers to PBMs and other managed care entities. Draft regulations recently have been proposed by the agency (FDA, 1995). PBM firms felt that this would lead to less timely dissemination of information and also would constrain potentially valuable sources of information that do not conform to FDA concepts of evidence regarding costeffectiveness (i.e. the need to perform two well-controlled studies for C-E claims). These issues are discussed further below.

\section{TREATMENT GUIDELINES, STEP PROTOCOLS AND DISEASE MANAGEMENT}

\section{Treatment guidelines for unique therapies}

The cost-effectiveness evaluation of unique new therapies is likely to be a central concern in the future for both PBMs and pharmaceutical firms. Pharmaceutical firms are now devoting a much larger share of their R\&D budgets to the search for drugs with unique therapeutic effects. They seek to command premium prices for these drugs on the basis of their advances for patient well-being and/or their savings in overall system costs. This underlies the strong interest of major pharmaceutical firms in health economic studies of these types of drugs.

At the same time, PBM firms are currently moving to risk sharing or even capitation arrangements with their larger clients. This makes them increasingly concerned with the costs and benefits of new therapies that lead to rapid growth in drug expenditures. For example, several PBM firms mentioned

*As indicated above, PBMs in alliance with several pharmaceutical firm partners are attempting to develop disease management programs for a number of pharmaceutical-intensive disease categories. These activities are considered below. the desirability of seeing cost-effectiveness and costbenefit evaluations of new drugs like Immitrex, an important recent advance in the treatment of migraine, that increases the expected drug costs of treating this condition.

As discussed above, new pharmaceuticals that offer significant therapeutic advances over existing therapies are currently admitted to the formularies of PBMs by the P\&T Committee. However, PBMs will make recommendations concerning their optimal utilization in guidelines disseminated to physicians. In some cases, PBMs may recommend to their clients that expensive new drugs be subjected to prior authorization.

One case example that we focused on in our discussions with PBM firms was the drug misoprostol (Cytotec). It is indicated for prophylaxis in individuals at high risk for developing complications from NSAID-induced gastric ulcers. This drug has no close substitutes. It has been the subject of several cost-effectiveness analyses (for a survey, see Barradell et al., 1993).

All of the PBMs we surveyed included misoprostol on their formulary and none of them subjected it to prior authorization. However, they recommended its use only for patients at high risk for developing complications from NSAID use (e.g. the elderly, those with concomitant debilitating diseases and those with prior history of gastric ulcers). This is generally consistent with the finding of C-E studies on misoprostol (Barradell et al., 1993). When queried, the PBM firms indicated that their guidelines for misoprostol were based on the medical decisions of their P\&T Committees. In this situation, the C-E studies were viewed as providing useful background support for decisions of the P\&T Committee.

In contrast to the PBM treatment of misoprostol, all of the PBM firms we surveyed targeted human growth hormone for prior authorization. Since this is a very expensive drug $(\approx \$ 25,000$ per year $)$, PBMs wished to insure that its use was confined to approved indications. Several of the other biotech drugs were also earmarked for prior authorization.

\section{Step protocols}

At the present time, most PBMs have disease treatment "guidelines" that are provided to participating physicians along with the drug formularies. These guidelines often take the form of step protocols in which older, cheaper drugs are the first-line therapies. Except for a few tightly controlled HMO clients, these guidelines are voluntary and educational in nature. While PBMs engage in a variety of incentive measures to influence drug selection within a particular chemical class of drugs, they are relatively passive in their approach to treatment guidelines. The choice of treatment regimen continues to be largely at the physician's discretion.* 
We investigated how treatment guidelines are developed and found that PBMs tend to follow very closely the recommendations of national commissions, government agencies and leading medical associations. For example, their guidelines often reference the Joint National Committee on Detection Evaluation and Treatment of High Blood Pressure, the National Cholesterol Education Program Expert Panel, the guidelines of the Association for Health Care Policy Research (AHCPR) and so forth. Leading medical texts and recent journal articles are also frequently cited in the formulation of these guidelines.

In our analysis of PBM guidelines, we decided to focus in particular on blood cholesterol reduction as a case study. This is an interesting case because a major new class of drugs have been introduced since the late 1980 s- the HMG-CoA reductase inhibitors or "statins". These drugs offer some significant therapeutic advantages compared to older therapies, particularly in the reduction of side effects. However, they are also considerably more expensive than some older competing therapies like nicotinic acid which are available generically. Cholesterol reducers have also been the subject of several cost-effectiveness studies in recent years.

We found the guidelines used by PBMs in the case of the treatment of high blood cholesterol have been strongly influenced by the Expert Panel of the National Cholesterol Education Program (NCEP).

*Between this initial report in 1988 and the revision of the NCEP guidelines in 1994, a number of cost-effectiveness studies were undertaken of cholesterol-reducing agents (Martens et al., 1989; Schulman et al., 1990; Hay et al., 1991; Goldman et al., 1991). They are essentially modeling studies which combine cost information from various sources with epidemiological data from the Framingham Heart Study. While these studies have been criticized for methodological shortcomings, they also have received considerable attention in academic and policy circles. A general finding which emerges from these analyses is that the cost to produce health benefits are lowest for groups with high nearterm risk for CHD. This is consistent with the NCEP. targeted approach for drug treatment and the recommendation for more aggressive treatment of secondary prevention in the 1994 guidelines. In addition, the newer HMG-CoA reductase inhibitors had more favorable C-E ratios than the bile sequestrant agents in a few studies that made comparative evaluations (see, for example, Coyle and Drummond, 1993).

†This study involves a prospective randomized trial undertaken at Southern California Kaiser Permanente in which half of the patients were randomized to the prior 1988 NCEP step therapy drug guidelines and the other half received Mevacor (Lovastatin) as their firstline drug therapy. The study seeks to approximate conditions of normal clinical practice at Kaiser with regard to provider and patient compliance and drug reimbursement terms. This type of study should address some of the concerns raised above on the need for C-E studies to examine patient compliance costs and overall effectiveness under real world practice settings in order to be persuasive to providers.
The first report of the NCEP was issued in 1988 (NCEP, 1988). It provided recommended targeted blood cholesterol levels for preventive treatment tied to a person's risk factors for coronary heart disease (CHD). The panel also recommended a stepped therapy approach consisting of first dietary therapy, then drug treatment with a bile sequestrant agent or nicotinic acid, and finally with a statin, only if cholesterol goals were not achieved with alternative therapies. The rationale for the use of bile sequestrant agents and nicotinic acid as firstline drug therapies related to their demonstrated long-term safety compared to the statins. At the same time, however, the statins were noted as highly effective in lowering LDL cholesterol and had a more favorable profile of side effects than the older therapies.

The 1994 NCEP panel made some significant changes in the guidelines (NCEP, 1994). First, the 1994 guidelines recommend a more aggressive drug treatment strategy in the case of secondary prevention. This is consistent with the results of C-E studies that are cited in the report.* In addition, the guidelines change the step protocol approach previously advocated. In the new guidelines, the statins along with bile acid sequestrants and nicotinic acid are classified as "major drugs" for treating cholesterol reduction. The report lists relative advantages and disadvantages of these alternative therapies, but does not make any recommendations concerning which should be the drugs of choice. This is an issue on which the panel apparently would like to see more research.

Most PBMs that we surveyed have adopted the new guidelines and now list the statins among the first-line drug therapies in their own guidelines. But not all have done so. A new study by Oster et al. (forthcoming) should provide further useful information to PBMs and other care providers in this regard. It examines the relative cost-effectiveness of a step protocol approach vs the use of an HMG$\mathrm{CoA}$ reductase inhibitor as the first-line agent, under the conditions of normal clinical practice at a major HMO. $\dagger$

The main lesson that emerges from our cholesterol case study is that the national consensus guidelines have exerted a strong influence on PBM decision processes, and these consensus guidelines in turn have been at least partially responsive to the cost-effectiveness studies performed to date on this issue. There are of course several other major therapeutic areas characterized by a range of newer and older drug treatment options (e.g. hypertension and depression) in which cost-effectiveness studies could also play a very useful role. It will be important to ascertain where stepped care based on lower cost medicines as the first option represent a cost-beneficial approach and where it leads to a sub-optimal result. This is likely a central issue in the appli- 
Table 7. Common disease management targets

\begin{tabular}{|c|c|c|c|c|c|}
\hline & Asthma & Diabetes & Cardiovascular & Depression & Gastrointestinal \\
\hline DPS & $\mathrm{x}$ & $\mathrm{x}$ & $\mathrm{x}$ & $\mathrm{x}$ & \\
\hline Medco & $x$ & $\mathrm{x}$ & $\mathrm{x}$ & $\mathrm{x}$ & $x$ \\
\hline PCS & $x$ & $\mathrm{x}$ & $x$ & $\mathrm{x}$ & $x$ \\
\hline \multicolumn{6}{|c|}{ Pharmacy } \\
\hline Gold & $x$ & $\mathrm{x}$ & $\mathrm{x}$ & & \\
\hline \multicolumn{6}{|l|}{ Value } \\
\hline Health & $x$ & $x$ & $\mathrm{x}$ & $\mathrm{x}$ & $x$ \\
\hline
\end{tabular}

Source: Interviews with five surveyed PBMs.

cation of pharmacoeconomic and health economic studies for the foreseeable future.

\section{Disease management}

As discussed above, the leading PBMs see disease management as a major area for future growth. Table 7 shows some of the principal disease management targets of the five leading PBMs. They have initiated various programs targeted at several high cost chronic diseases (diabetes, asthma, depression, etc.). In contrast to their treatment guidelines discussed in the last section, these programs will entail very active intervention strategies.

A motivating factor behind the PBM movement toward disease management is the premise that medical costs in many diseases are concentrated in a small portion of the affected population. Furthermore, many of these costs are also associated with out-of-control cases that have greater emergency room use, hospitalization and other resource-intensive interventions. Hence, if one can identify high risk patients and intervene in advance to change their treatment and compliance patterns, there are potentially large cost savings as well as improved quality of care for patients. The choice of diseases like diabetes and asthma have been selected as disease management targets by all of the leading PBMs because they fit the model presented above and offer large potential economic opportunities to those firms that can successfully implement disease management programs.

A comprehensive disease management program involves data integration, analysis, implementation and outcomes evaluation. Currently PBMs have very comprehensive prescription drug information and potential access to other medical cost data through their clients. A major activity at the present time is the integration of data with compatible coding systems from physicians, laboratories, home health care and hospitals with prescription drug expenditures. Some firms are analyzing health outcomes with the data sets of HMO clients that are already integrated.

A typical project might involve a retrospective analysis of integrated medical data bases for asthma patients. The objective would be to identify factors leading to above-average treatment costs (e.g. inappropriate drug treatment or poor patient drug compliance in certain demographic groups) and to develop management techniques to change these adverse outcomes (e.g. a targeted monitoring and compliance program using pharmacists, nurses, case managers, physician education, etc.).

Implementation of a disease management program for asthmatic patients would concentrate on controlling the disease, thereby reducing costly emergency department visits and hospitalizations. Appropriate patient and provider education would lead to improved compliance and correct administration of inhaled bronchodilators, as well as proper use of anti-inflammatory agents such as corticosteroids. An asthma management program should also involve environmental and lifestyle modification. This would include avoidance of triggers and allergens. Prevention of asthma exacerbations is a principle objective of a successful disease management program, as documented in the Expert Panel Report on Guidelines for the Diagnosis and Management of Asthma (U.S. Department of Health and Human Services, 1991). Outcomes analysis is also an essential component of these programs in order to demonstrate their effectiveness and cost savings.

Similarly, a disease management program for hypertension would develop protocols to implement primary prevention, screening, and treatment programs. For initial drug therapy, PBMs will need to determine if newer products with higher direct acquisition costs, such as calcium channel blockers and angiotensin II receptor antagonists, are more cost-effective than traditional antihypertensive medicines such as diuretics and beta-blockers. The key to a successful hypertension program, however, also involves individualized patient regimens, reinforcement and continuous care. social support, and the collaboration of various health care professionals. as described by the Joint National Committee on Detection, Evaluation, and Treatment of High Blood Pressure (Gifford et al., 1993). Thus, PBMs will be challenged with not only selecting the appropriate drug therapy for patients, but also will need to focus on lifestyle modification including tobacco avoidance, weight reduction, moderation of alcohol and dietary sodium intake, and promotion of physical activities. These may be accomplished through patient newsletters, direct patient contact, or innovative patient education programs. 
Another aspect of disease management involves integrated management of medical and pharmaceutical care through an electronic on-line network at the point of medical care. While virtually all pharmacies currently participate in on-line networks, only a small percentage of physician practices do. PCS, which was a prime mover in getting pharmacies on-line, is also taking the lead in this regard with medical practitioners. Their vision for the future is "electronic coordinated care" with directto-physician electronic communications for guiding drug treatments in particular diseases.

The interest of PBMs in disease management implies an increased attention on their part to outcomes analysis and cost-effectiveness studies. The leading firms have been building a sizeable in-house expertise with several Ph.D.s and other professionals in this area. They have begun a number of their own cost-effectiveness analyses, both independently and in conjunction with academic centers, pharmaceutical firms and HMO partners. Their activities can be expected to grow over time as disease management becomes a more central focus of PBMs and other managed care organizations.

Over the long run, there are a number of interesting industrial organization-type research issues concerning the emerging role of PBMs in disease management. These include the comparative advantage of PBMs in disease management, how the market will be structured, and how firms will be compensated for performing these activities. These issues remain important topics for future work.

\section{SUMMARY AND POLICY CONSIDERATIONS}

Pharmacy benefit management companies have evolved rapidly during the 1990 s from claims processors and pharmacy networks to the management of drug product selection using formularies and related incentive measures. The main economic drivers of this process have been generic substitution and manufacturer discounts for formulary listings from among closely substitutable drugs. The emphasis has been on lowering drug costs rather than taking a broader perspective on cost-effective treatments.

Although PBMs have demonstrated that they can achieve significant cost savings in the drug budgets of employers and managed care entities from formulary-type interventions on a carved-out basis, over the long run management of drug benefits as a

*As many researchers have pointed out, a randomized controlled trial study design provides the highest degree of internal validity, but it also leaves open many questions concerning external validity. This reflects several factors-- the nature of patient and provider populations participating in trials, the presence of protocol-driven costs and outcomes, the use of extraordinary measures to ensure patient compliance. the time horizon used in trials and so forth. separate component, in isolation of other health care activities, has important limitations. By its very nature, it cannot capture the larger cost efficiencies potentially available from an integrated treatment approach to medical problems and diseases.

Pharmacy benefit management companies appear to recognize that the market is likely to evolve into more comprehensive treatment approaches to drug management in the near future. The leading PBM firms have been investing heavily in disease management programs. A key first step in this process is development and analysis of data bases and outcomes information. This process is already underway with PBMs performing a variety of modeling efforts and cost-effectiveness analyses of patient data in several target disease areas (asthma, diabetes, depression, etc.). This is likely to expand over time. PBMs can be expected to do their own costeffectiveness studies, but also participate in a variety of cooperative ventures with drug manufacturers, HMOs and academic bodies using a variety of methodological approaches.

The growth of PBMs can be viewed as part of a broader evolutionary selection process currently taking place in the health care system. Though a trial and error process, the market is currently searching for organizational structures that can provide increased cost efficiency and other performance improvements. In this dynamic evolutionary market, it is relevant to ask what constraints should be placed on information exchanges between the different parties in the market. In a set of draft resolutions, the FDA has proposed that pharmacoeconomic information provided by manufacturers to prescribers and purchasers be treated as part of a drug's labeling. Furthermore, it proposes that any cost-effectiveness claims be supported by two adequate and well-controlled studies similar to what is currently required in support of a drug's safety and efficacy (FDA, 1995).

Pharmacy benefit management companies have indicated that they want timely C-E studies with "real world" medical practice environments and comparators. In the current market-driven environment, a number of methodological approaches are emerging to deal with these issues including modeling studies as well as experimental and quasi-experimental designs. The FDA approach would constrain the availability of information from many studies which are critical to the evaluation of costeffectiveness in real world settings.*

Another set of issues relate to the effects on $R \& D$ costs and timeliness of FDA requirements. If manufacturers have to do multiple full-scale randomized control trials and receive FDA approval before making cost-effectiveness claims to managed care entities, this would not only increase $R \& D$ costs but also would delay the availability of useful information to decision-making entities. Cost-effectiveness decisions are a moving target, and decision- 
making should have the benefit of information in as timely a fashion as possible. In addition, the higher costs would likely mean less information for many NCEs with smaller market sales.*

Based on our interviews with leading PBMs, it is clear that these organizations also have considerable in-house expertise in pharmacoeconomics and outcomes analysis. Some of the leading PBMs have a healthy skepticism of manufacturer-generated studies and can put their own weights on the claims of manufacturers and the quality of journal publications where this information is published. Indeed, PBMs are currently doing various modeling analyses of particular diseases like asthma or diabetes that are exactly the kind of studies for which manufacturers would not be allowed to distribute information. This is not a desirable regulatory outcome.

Further research on the costs and benefits of the government regulation of cost-effectiveness claims by manufacturers to managed care entities should be a high priority area for future work. It is significant that the proposed FDA regulations are not something being sought by any of the PBMs that we interviewed. It is doubtful that these regulations could pass a cost-benefit test from either a private or societal perspective. To the extent that a thirdparty review of cost-effectiveness claims is desirable, market alternatives to government regulation should be explored. A possible model worth considering in this regard is the independent auditing of financial data by publicly certified accountants.

\section{REFERENCES}

Barradell, L. B., Whittington, R. and Benfield, P. (1993) Misoprostol: pharmacoeconomics of its use as prophylaxis against gastroduodenal damage induced by nonsteroidal anti-inflammatory drugs. PharmacoEconomics 3(2), 140-173.

Blissenbach. H. F. (1993) Pharmaceutical services in managed care. In The Managed Health Care Handbook, 2nd edn, ed. P. Kongfvedt, pp. 142-160. Aspen, Gaithersburg, MD.

Coyle, D. and Drummond, M. (1993) Does expenditure on pharmaceuticals give good value for money: current evidence and policy implications. Health Policy 26, 5575 .

U.S. Food and Drug Administration (FDA) Principles for the review of pharmacoeconomic promotion. Draft manuscript, FDA.

Gifford, R. W.et al. (1993) The fifth report of the Joint National Committee on Detection, Evaluation, and Treatment of High Blood Pressure. Archives of Internal Medicine 153, 154-181.

Goldman, L., Weinstein, M. C., Goodman, P. A. and Williams, L. W. (1991) Cost-effectiveness of HMG-CoA reductase inhibitors for primary and secondary preven- tion of coronary heart disease. Journal of the American Medical Association 265(9), 1145-1151.

Grabowski, H. G. (1988) Medicaid patients' access to new drugs. Health Affairs Winter, 102-114.

Grabowski, H. G. (1995) Price and profit control, new competitive dynamics and the economics of innovation in the pharmaceutical industry. In Industrial Policy and the Pharmaceutical Industry, ed. A. Towse, pp. 77-91. Office of Health Economics, London.

Grabowski, H. G. and Vernon. J. M. (1992) Brand loyalty entry and price competition in pharmaceuticals after the 1984 act. Journal of Law and Economics 35, 331-350.

Grabowski, H. G. and Vernon, J. M. (1994) Returns to $\mathrm{R} \& \mathrm{D}$ on new drug introductions in the 1980s. Journal of Health Economics 13, 383-406.

Grabowski, H. G. and Vernon, J. M. (1996) Longer patents for increased generic competition: the WaxmanHatch Act after one decade. PharmacoEconomics 10(2), $110-123$

Hay, J. W., Wittels, E. H. and Grotto, A. M. (1991) An economic evaluation of lovastatin for cholesterol lowering and coronary artery disease reduction. American Journal of Cardiology 67(9), 789-796.

Health Strategies Group (1995) Pharmacy Benefit Management. HSG, Palo Alto, CA.

Martens, L. L., Rutten, F. F. H., Erkelens, D. W. and Ascoop, C. A. P. L. (1989) Cost-effectiveness of cholesterol-lowering therapy in the Netherlands: simvostatin versus cholestramine. American Journal of Medicine 87(4A), 545-585.

Moore, W. J. and Newman, R. J. (1992) U.S. Medicaid drug formularies: do they work. PharmacoEconomics 1(1), 28-31.

National Cholesterol Education Program (1988) Report of the expert panel on detection, evaluation and treatment of high blood cholesterol in adults. Archives of Internal Medicine 148, 36-69.

National Cholesterol Education Program (1994) Second report of the expert panel on detection, evaluation and treatment of high blood cholesterol in adults. Circulation 89(3), 1333-1445.

Oster, G., Borok, G. M., Menzin, J., Heyse, J. F. Epstein, R. S., Quinn, V., Benson, V., Diedl, R. J. and Epstein, A. M. (1996) Cholesterol-Reduction Intervention Study (CRIS): randomized trial to assess effectiveness and costs in clinical practice. Archives of Internal Medicine.

Schulman, K. A., Kinosian, B., Jacobson, T. A., Glick, H., Willian, M. K., Koffer, H. and Eisenberg, J. M. (1990) Reducing high blood cholesterol with drugs. Journal of the American Medical Association 264(23). $3025-3033$.

Shaw, H. K. (1992) Catalysts for Change: The Impact of Generic Drugs on the Pharmaceutical Industry Spectrum. Report 38-8, Decision Resources, Burlington, MA.

Treppell, J. (1994) Generic Industry Drug Interview. Kidder Peabody, New York

U.S. Department of Health and Human Services (1991) Guidelines for the Diagnosis and Management of Asthma. National Asthma Education Program Expert Panel Report. NIH No. 91-3042. Bethesda, MD

U.S. General Accounting Office (1995) Early Results on Ventures with Drug Manufacturers. GAO/HEHS-96-45. General Accounting Office.

\footnotetext{
*Grabowski and Vernon (1994) have found that the distributions of returns to new drugs is highly skewed. Their work suggests that it would be unprofitable to undertake separate costly C-E studies for the majority of new entities.
} 